\title{
Pulsed-Pressure MOCVD Science, Materials and Technology
}

\author{
Susan P. Krumdieck \\ Department of Mechanical Engineering, University of Canterbury, \\ Christchurch, New Zealand
}

Pulsed pressure processing from liquid MOCVD precursors has been studied over the past ten years. Extending the capabilities of CVD to applications like thermal barrier coatings, Solid Oxide Fuel Cells, and bio-integration coatings was the motivation for the original innovative concept. The systems engineering research has focused on development of a low-cost, high throughput method capable of providing high quality, uniform ceramic films on metal, ceramic or glass objects with complex 3-D geometry. The MOCVD research has focused on using low-cost, low-hazard precursors to deposit the desired materials with the required properties. This paper reviews the fundamentals of the PPMOCVD approach, the experience with precursors and thin films, and the advances of the system engineering toward several commercial applications.

\section{Introduction}

Over the past thirty years a wide range of CVD processing methods have been developed, each with its own acronym. The fundamental CVD process is essentially the same, regardless of whether it was carried out at atmospheric pressure, ultra-high vacuum, or whether the reaction energy was supplied by plasma or ultra violet radiation. Vapor phase chemical precursors undergo an activated reaction on a surface to deposit a solid material layer. The reason there are so many variations on CVD technology is that there is no possibility of direct control of the basic CVD process at the deposition surface. The details of the process technology establish the thermal and mass transport dynamics in the reactor, and thus are important determinants in the kinetics of the CVD process at the surface and ultimately the film material properties. The deposited film quality and uniformity are usually better if the rate-controlling step is the surface reaction kinetics. The growth rate is faster in higher pressure and higher temperature processes where the diffusion mass transfer rate to the surface is the rate limiting step, but the uniformity is difficult to control due to viscous flow structures, and quality may be a challenge due to gas phase particle formation. CVD technology development also involves other considerations including the affordability, process throughput, integration with other manufacturing steps and maintenance requirements (1).

CVD technology is usually discussed in terms of the benefit and issues trade-offs. For example, atmospheric pressure CVD (APCVD) has high growth rate and has been successfully incorporated into architectural float glass manufacturing at cost commensurate with the improved thermal and self-cleaning properties. Large, APCVD batch processes have also been developed for hardness layers on cutting tools. However, to achieve the quality and uniformity needed for electronics applications, low pressure 
processing (LPCVD and MOVPE) and atomic layer deposition (ALD) are used. The benefits of uniformity and quality control are traded-off against processing time and cost.

The processing technology reviewed in this paper was engineered in response to a set of challenging objectives; CVD quality, micro-scale conformality, macro-scale uniformity, low cost, rapid prototype and design for industrial production. The opportunity presented by new MOCVD precursors indicates possible new applications for thin ceramic films as functional or active layers for a range of products. For example, high temperature casting moulds wear out quickly due to thermal stresses, and are expensive to machine from high temperature alloys. A possible solution could be to have a thermal barrier coating (TBC) of zirconia on the mould. The zirconia film must have columnar microstructure and be at least $50 \mu \mathrm{m}$ thick. The coating would likely wear out with use and would need to be re-applied periodically. The mould would have a complex shape, and the cost of the TBC coating system would need to be less than the alternative of machining new molds. A new approach would be needed to realize MOCVD in the foundry workshop to re-coat moulds at low cost.

The Ultimate CVD Processing Challenge Produce ceramic layers on metal, glass or ceramic objects with both macro and micro-scale, three-dimensional features. The films must be uniformly deposited with control of microstructure, and must have high quality without particle or reactant product contamination. The layer thickness must be easily controlled between $10 \mathrm{~nm}$ and $50 \mu \mathrm{m}$. The process equipment and running cost must align with the product.

The Pulsed-Pressure MOCVD research program has been aimed at development of the technology for low-cost coatings and functional films for a wide range of new applications. The fundamental operating principle for PP-MOCVD involves the mass transport dynamics due to rapid expansion of precursor into the evacuated reactor volume without carrier gas (2). Expansion is a non-continuum process, the result of which is equilibrium. Thus, expansion mass transport can produce uniform deposition conditions (3). Figure 1 shows an experimental apparatus used for expansion mass transport research with objectives to develop the relations for reactor design and process control.

This paper reviews the fundamental principles and research progress to-date in the development of the PP-MOCVD technology. The paper is organized into five sections presenting the different research and development projects carried out over the past ten years. The first section gives the details of the PP-MOCVD method and equipment, as well as the basic operating principles. The second section sets out the theory of PPMOCVD flow dynamics and process control for flow-field

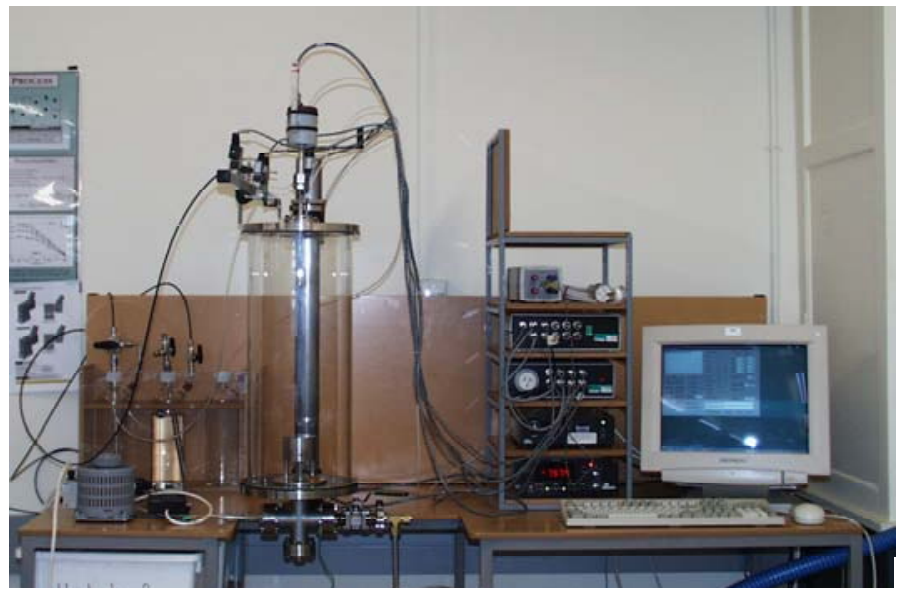

Figure 1. Experimental PP-MOCVD apparatus used for mass transport, system design and process control research, built in-house for less than $\$ 20,000$ US. 
uniformity. The next section reviews the recent work on numerical modeling of the unique flow conditions, and the implications for both liquid and gas pulsed injection. Experience with a range of materials deposited by PP-MOCVD is covered in the final section.

\section{The Pulsed-Pressure MOCVD Method}

The objective of PP-CVD is to gain control of the deposition parameters through sequential processing with engineering control of the precursor supply and reactor conditions. The design philosopy is focused on simplicity, low cost equipment and reliable operation. The precursor supply equipment is designed to provide a consistent and repeatable dose of precursor to the reactor during each pulse. The technology to-date has focused on cold-wall reactors to take advantage of the high efficiency and lower cost. Because the reactor is evacuated at the end of each pulse, the PP-MOCVD method should produce contaminant-free films even though the base pressure is provided by lower cost rotary vacuum pumps.

\section{PP-MOCVD Operating Theory}

The key to the method is the unique mass transport regime developed by both the rapid injection phase and the pump-down evacuation phase of each pulse cycle. The basic idea is that expansion of vapor to fill a vacuum must produce a uniform, well mixed condition in the reactor volume. Further, the expansion process is on a time scale much faster than the time for a viscous flow pattern to develop during the injection. The injection is short, and after it is shut off, the reactor is evacuated. As long as the injection pressure rise is fast enough, the operating pressure does not affect the uniformity of the expanded vapor mixture. As long as the pump-down time is long enough, the cycle will be repeated for each pulse. If the precursor adsorption and reaction rates are faster than the pump-down rate, conversion efficiency can be high. The gas diffusivity increases exponentially as the pressure decreases during the evacuation. Thus, the diffusion of the precursor to the surface will always be fast enough that concentration gradients will not develop throughout the reactor. The result of this operating regime is that the deposition process is not sensitive to the geometry and that the reactor and system design can be scaled up or down according to three simple scaling factors, injection time, pump-down time, and the pulse pressure rise $\left(P_{\max }-P_{\min }\right)$. Expansion regime operation therefore could allow low-cost process and equipment development for application of CVD research results to new products.

\section{$\underline{\text { Process Fundamentals }}$}

Details of the PP-MOCVD method and operating principle are shown in Figure 2. Each cycle begins with an instantaneous injection of a metered volume, $v_{p}$, of liquid precursor solution, via an ultrasonic atomizing nozzle, into an evacuated low pressure, $P_{\text {min }}$, reactor with volume, $V_{R}$. The ultrasonic nozzle supplied by Sono-Tek, can handle a range of flow rate, and produces a fine mist of droplets in the range of 2-30 $\mu \mathrm{m}$ diameter. The precursor solution flash evaporates due to the low pressure. The concentration, AB, of moles of liquid precursor $\mathrm{A}$ in a volatile solvent $\mathrm{B}$ can be accurately controlled by careful measurement in preparation. 


\section{Process Control \\ $N_{p}$ number of pulses \\ $t_{p}$ pulse cycle time \\ $P_{\max }$ peak pressure \\ $P_{\min }$ pump-down \\ $v_{p}$ liquid injection volume \\ $A B$ precursor concentration \\ $V_{R}$ reactor volume \\ $S_{R}$ evacuation speed \\ $\tau_{R}$ pump down time const \\ $T$ substrate temperature}

\section{Pulsing Operation Reactor Pressure Profile}

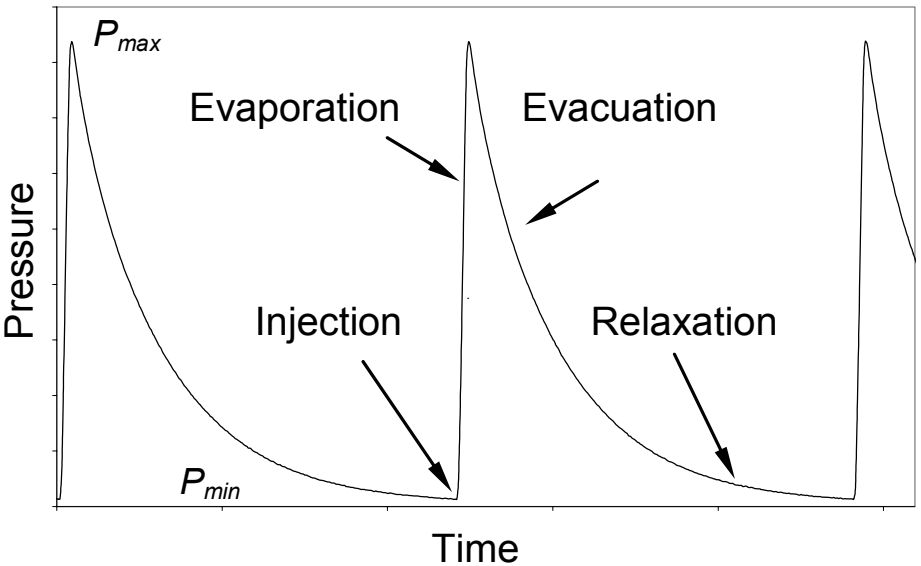

\section{Injection}

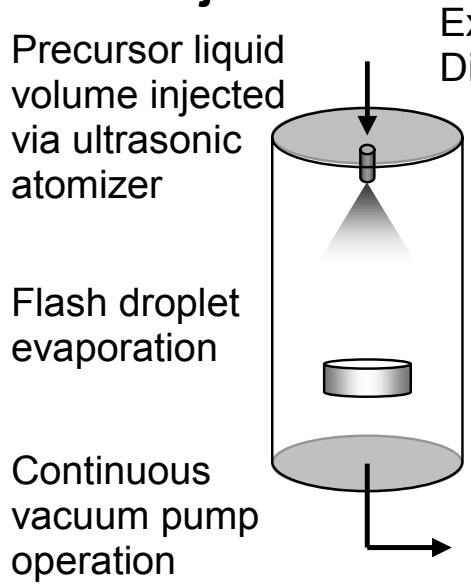

Molecular flux from uniform concentration, quiescent vapor to surface with increasing gas diffusivity during process

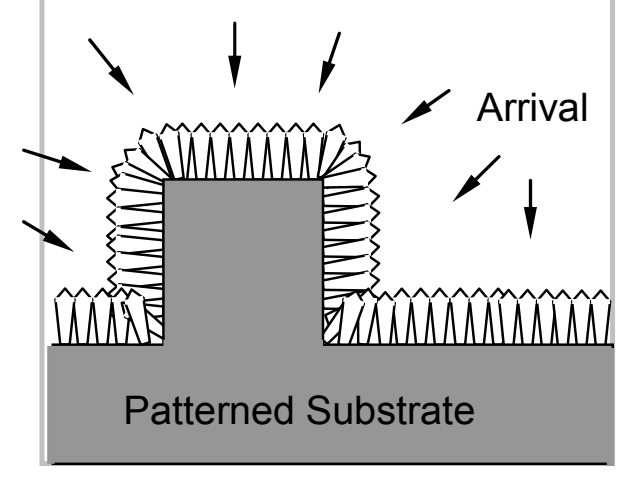

\section{Injection}

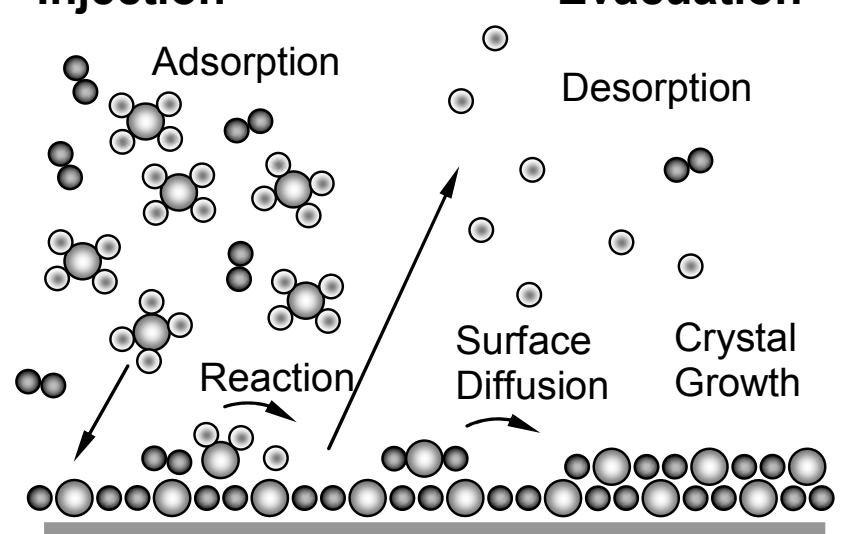

Figure 2. The key point of difference for PP-MOCVD from steady-flow CVD technology is the rapid change of pressure in the reactor which produces an expansiondominated mass transport field with uniform dispersal of the precursor and low sensitivity to reactor and substrate geometry. The pulsed operation facilitates rapid design and development of industrial-scale reactors. 
The volume of liquid precursor injected is accurately controlled by isolation in the metering loop of a low dead-volume 6-way valve commonly used to isolate and inject samples in gas-chromatography machines. Flash evaporation of the precursor results in a sharp pressure rise in the reactor to a maximum value, $P_{\max }$, which will depend on the injected volume and reactor volume according to the ideal gas law. As long as the maximum pressure is well below the vapour pressure of the liquid solution, $P_{v}$, the evaporation will be complete and can be considered "flash" evaporation. A solution of single or multiple metalorgainc precursors can be prepared with a compatible volatile solvent. As long as the precursor does not aglomerate in solution, a small, lowconcentration droplet injected into a vacuum well below the mixture vapor pressure will undergo supercritical phase change. This will effectively vaporize the precursor without heating and the risk of pre-mature decomposition or reaction. There are numerous advantages of direct liquid injection over heated bubblers and transport by carrier gas to the reactor. The vapor concentration above a mixture in a bubbler depends on the concentration and volatility of the constituents. Injecting the liquid directly allows control of the precursor concentration throughout the process. There are no problems of condensing in supply lines, although particulates can clog or damage liquid control valves.

PP-MOCVD from reactive gas precursors can also be accomplished. A pulse of gas from a high pressure source with volume, $V_{s}$, is injected through an oriface in a short burst. The length of time that the gas injection valve is opened to the high pressure source is the injection time, $t_{i}$. The mass of gas that enters through the orifice with cross section area, $A_{s}$, during the injection is usually determined by choked sonic flow due to the very high pressure gradient from the source, $P_{s}$, to the reactor vacuum according to the discharge coefficient, $C_{s}$. The mass injected during each pulse can be accurately controlled through simple pressure regulation on the source cyllinder and timing of the injection valve. The time constant for gas injection depends on engineering of the supply system, $\tau_{\mathrm{s}}=V_{s} / C_{s}$, while the time constant for liquid injection depends on the droplet evaporation time and is extremely short compared to the gas injection for the same pressure rise in the reactor.

The injection process is followed by reactor pump-down to complete one pulse cycle. The reactor is constantly evacuated by a vacuum system with pumping speed at the reactor outlet of $S_{R}$. The pump-down time constant is a function of the reactor volume and the evacuation speed, $\tau_{R}=V_{R} / S_{R}$. Viscous flow patterns will not develop in a vessel during pump-down unless there are small orifaces or constrictions between the main reactor volume and the exhaust outlet. The vapor in the reactor effectively experiences simple pressure reduction. Thus, the mass transport process during the pump-down phase of the pulse cycle is also an expansion process. Concentration gradients could form in the quiescent reactor during the pump-down phase if the adsorption rate at the heated surface was faster than the gas diffusion rate. However, this is not the case as the gas diffusion rate of species A in species B varies inversely with the total pressure. As the reactor total pressure decreases, the diffusivity increases and the fastest process in the reactor is the continuous driver for equilibrium through re-establishment of pressure and concentration uniformity (4). Finally, the removal of reaction products from the deposition zone and the reactor is another area where PP-MOCVD has benefits over conventional steady-flow CVD. During the pump-down process, the partial pressure of reaction products in the vapour is continuously reducing and the diffusivity is also increasing. Thus the reaction products are actively removed during the evacuation phase of the pulse cycle. 
Equation 1 gives the pressure during gas injection derived from mass balance and assuming choked inlet flow condition. Equation 2 gives the pressure during the pumpdown phase. The molecular arrival rate at a surface in the reactor volume, assuming a well-mixed condition, is given by rarefied gas dynamics (5). Assuming that the injection is either instantaneous flash evaporation or short pulse gas injection, $t_{i}<0.1 \tau_{\mathrm{s}}$, and that the vapour concentration is constant, the molecular arrival rate during a pulse, $J_{P}$, can be found by integrating the pressure over the pump-down phase as shown in Equation 3.

$$
\begin{array}{r}
\frac{P(t)-P_{\min }}{P_{s}}=\frac{V_{s}}{V_{R}}\left(1-\exp \left(\frac{-t}{\tau_{s}}\right)\right) \quad\left(\text { for } 0 \leq t \leq t_{i}\right) \\
\frac{P(t)-P_{\min }}{P_{\text {max }}-P_{\min }}=\exp \left(-\frac{t}{\tau_{R}}\right) \quad\left(\text { for } t_{i} \leq t \leq t_{p}\right) \\
J_{P}=\left\{\frac{A B \cdot N_{A}}{\sqrt{2 \pi M R_{o} T}}\right\}\left[P_{\min _{p}} t_{p}+\tau_{R}\left(P_{\max }-P_{\min }\right)\left(1-e^{\left.\left.-t_{p} / \tau_{R}\right)\right]}\right.\right.
\end{array}
$$

A non-dimensional processing parameter for exposure to precursor vapor over each pulse, $E^{*}$, is indicated by Equation 3 and found by dimensional analysis in terms of the processing variables as given in Equation 4. The precursor exposure and substrate temperature are the key processing variables in PP-MOCVD that affect the material growth rate and properties. The pulse timing in relation to the equipment time constants are the key design parameters as will be discussed in the next section.

$$
E^{*}=\frac{A B[\mathrm{~mol} \%] \cdot v_{s}\left[\mathrm{~mm}^{3}\right] \cdot\left(P_{\max }-P_{\min }\right)\left[\mathrm{kg} \cdot \mathrm{s}^{-1} \mathrm{~m}^{-1}\right]}{V_{R}\left[\mathrm{~m}^{3}\right] \cdot P_{v}\left[\mathrm{~kg} \cdot \mathrm{s}^{-1} \mathrm{~m}^{-1}\right]}
$$

\section{$\underline{\text { PP-MOCVD Technology }}$}

The pulsed-pressure operation can be applied for either direct liquid injected precursor solutions or gas precursors. Both possibilities have been studied, with similar findings regarding the uniformity of the mass transport, the process control and system design (6). All PP-MOCVD systems to-date have been based on the system configuration shown in Figure 1. Sono-Tek has provided small research systems to the market capable of deposition on $5 \mathrm{~cm}$ wafers from liquid precursors. All reactors have been cold-wall vertical tube configuration with the precursor injection at the top, the heated substrate stage at the base, and exhaust through the bottom flange. The research reactors are made from tempered Pyrex glass with stainless steel flanges sealed with viton O-rings at either end. A range of reactor diameters and heights have been used. The reactor vessels for liquid precursor systems used for materials research are typically 75-85 mm diameter with length between 350 and $450 \mathrm{~mm}$. Gas injection systems have been constructed for mass transport research and diamond deposition, with the reactor vessels ranging in size up to $210 \mathrm{~mm}$ diameter and $530 \mathrm{~mm}$ high.

Reactor pressure for gas injection experiments is measured by capacitance manometers (MKS BARATRON) with response time constant less than $20 \mathrm{msec}$ and accuracy of $0.25 \%$. Normally at least two manometers are needed to measure the upper 
and lower range of the pulse pressure. The materials deposition system uses a convectionenhanced pirani gage (MKS 947). The heaters are made in-house from wound kanthal wire with operating temperature to $1200{ }^{\circ} \mathrm{C}$, potted in alumina clay and fired at $1100{ }^{\circ} \mathrm{C}$. The heaters can provide deposition temperatures up to $720{ }^{\circ} \mathrm{C}$. Further details of the various experimental apparatus used for particular materials research and for mass transport investigations are given in the various publications discussed in detail in the following sections. All of the systems use similar exhaust and injection systems briefly described below.

The exhaust systems make use of two stage rotary vaned pumps. These pumps are capable of base pressures in the range of $1 \times 10^{-4} \mathrm{mbar}$ and are at the lower end of the cost range for vacuum pumps. The small materials reactor is evacuated by one pump with a liquid nitrogen fore line trap and shut-off valve to isolate the reactor from the exhaust system after deposition experiments while the heater is cooling. The exhaust system for the larger reactor gas injection experiments was varied to produce different exhaust rates by adding up to three vacuum pumps in parallel. The reactor volume and pump speed determine the pulse cycle time for the reactor pressure to return to the base pressure. In the smaller materials research reactor the pulse cycle time is 10 seconds, whereas in the largest reactor the cycle time was 40 seconds with one pump.

The injection system design is key to the simplicity and low cost of the PP-MOCVD technology. Delivering a controlled flow of liquid to a reactor vessel at low pressure is a challenge. Normally, we move liquid by creating a high pressure with a pump, or by pressurizing a supply ampoule with inert gas. In delivering liquid to a vacuum, however, the liquid would get sucked into the reactor and bubbles would form in the supply tubes as the liquid boils off due to the low pressure. The Jipelec pulsed liquid injection system addresses this by using a high frequency injection valve that opens into a heated evaporation chamber (7). The injection pulses are so small that the reactor pressure is essentially constant, and a flow of carrier gas is used to transport the vapor to the reactor. In contrast, the PP-MOCVD inlet injection system does not use a carrier gas, and the pulses create a dramatic pressure change in the reactor. Each injection cycle has two stages. In the first step, an injection volume is filled with precursor solution from the pressurized ampoule. A slight overflow to a drain container ensures that the injection volume is completely filled. The injection volume is a length of tubing fitted into the supply loop of a gas-chromatograph 6-way sample injection valve. A small inert gas charge is filled during the first stage as well. In the second stage, the gas charge is opened to the injection volume tube to push the liquid into the reaction chamber through the ultrasonic atomizing nozzle. This rapid injection results in the nearly instantaneous pressure rise to the maximum or peak pressure.

\section{Experimental Methods for Flow Field Uniformity}

Flow field concentration uniformity leads to a uniform 3-D deposition. The naphthalene sublimation technique was used to measure the mass transport uniformity in the chamber. Small cylinders of naphthalene were cast and suspended from a slender wire frame throughout the volume of the reactor. The specific sublimation rate of each cylinder depends on temperature, pressure and the convection mass transport field. Uniform pressure, convection and concentration fields throughout an isothermal reactor, would result in the same specific sublimation rate for all the naphthalene cylinders. 
The specific sublimation rate, $\dot{S}_{i}\left[\mathrm{mg} \cdot \mathrm{s}^{-1} \cdot \mathrm{mm}^{-2}\right]$, for each naphthalene cylinder is easily calculated from the mass loss divided by that cylinder's surface area, and the total experiment time. The measurement accuracy is $\pm 0.02 \mathrm{mg} / \mathrm{sec} \cdot \mathrm{mm}^{2}$ so the experiment time was set so that the error in the mass sublimation measurement from loading and weighing was less than $2 \%$. The uniformity can then be evaluated for different operating conditions at different pressures, by normalizing the deviation data with the total sublimation. The convective uniformity, $U$, for $M$ naphthalene samples placed at positions throughout the reactor taking into account not only the average sublimation $\overline{\dot{S}}$ but also the total magnitude of the sublimation is given as:

$$
U=\frac{\sum_{i=1}^{M} \dot{S}_{i}-\sum_{i=1}^{M}\left|\dot{S}_{i}-\overline{\dot{S}}\right|}{\sum_{i=1}^{M} \dot{S}_{i}}=1-\frac{\sum_{i=1}^{M}\left|\dot{S}_{i}-\overline{\dot{S}}\right|}{\sum_{i=1}^{M} \dot{S}_{i}}
$$

If all samples have the same specific sublimation rate, regardless of the absolute magnitude, then the uniformity will have a value of unity. During a particular test, if some samples have very high sublimation while others sublime slower than the average, then the uniformity will have a low value. In principle, the range for the uniformity measure would be $0 \leq U \leq 1$, although several orders of magnitude difference in specific sublimation is needed to get vanishingly small uniformity. The results shown in Figure 3 indicate the operation and design rules that $t_{p} / \tau_{\mathrm{R}} \geq 4$ and $t_{i} / \tau_{\mathrm{s}} \leq 0.1$.

\section{Naphthalene Sublimation Uniformity for Various Reactors and Injection Conditions}

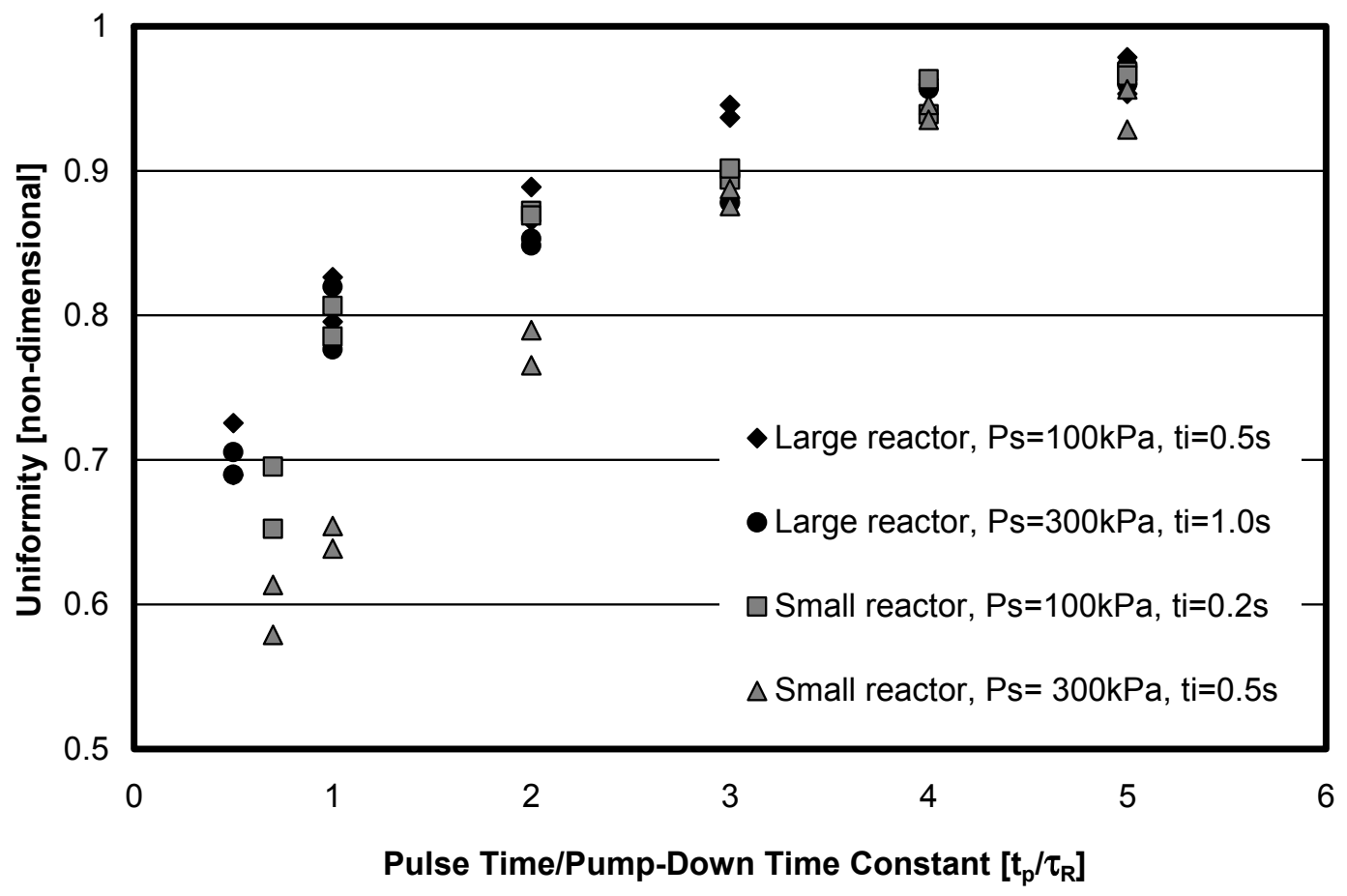

Figure 3. Results of naphthalene sublimation experiments for different size reactors, showing that mass transport uniformity requires sufficiently long pump-down time and short injection time. The uniformity for steady flow was typically in the range $0.4-0.5$. 


\section{Numerical Modeling of PP-MOCVD Processes}

Numerical modelling of the non-continuum, un-steady flow required development of a new approach $(8,9)$. A short term gas jet injection into the low-pressure reactor was shown to behave as an under-expanded jet and the turbulent mixing involved generates a well-stirred quiescent reactor condition at the end of the injection (10). Assuming a sticking coefficient of unity and a Monte Carlo simulation of the gas dynamics during the pump-down phase, the possibility of high precursor conversion efficiency was demonstrated (11). A new numerical method termed "Quiet Direct Simulation" has been developed to model the droplet evaporation and more complex internal geometries for new industrial-scale reactors (12).

\section{Materials}

Several ceramic materials have been studied in depth in order to characterize the relationship between processing parameters and growth rate and morphology. $\mathrm{TiO}_{2}$ was deposited from TTIP on nickel pieces demonstrating Arrhenius growth rate with temperature and precursor conversion efficiency above $90 \%$ in what is normally the diffusion controlled region (13). Linear dependence of growth rate on precursor concentration further indicated that the rate controlling step in the mid temperature ranges could be mass transport rather than diffusion (14). $\mathrm{TiO}_{2}$ was used to study the conformality of thin films on nano-scale and micro-scale features on patterned substrates (15). Figure 4 shows some recent results of $\mathrm{TiO}_{2}$ deposition over lithographed trenches on silicon nitride substrates. At very high exposure, the tops of features show accelerated deposition rate compared to the trenches as seen in Figure 4(d). Moderate and low precursor concentration produced uniform coverage as in Figure 4(a) and 4(b). The structures are similar to those reported by Akiyama et al. for diffusion controlled growth in a concentric flow horizontal tube reactor with a high degree of uniformity (16).

This capability to control the morphology through the processing parameters was used to produce sealing layers of yttria-stabilized-zirconia deposited on porous solid oxide fuel cell electrodes (17). The liquid injection of a solution containing both yttrium methoxyethoxide and zirconium 2-methyl-2-butoxide precursors in toluene was demonstrated to produce YSZ, with film growth rate $7.5 \mu \mathrm{m} \cdot \mathrm{h}^{-1}$ at cost of $\$ 0.50$ US per $\mu \mathrm{m} \cdot \mathrm{cm}^{2}$ (18). The unique aspect of PP-MOCVD, that the deposition process can be stopped after one or several pulses, was used to investigate the nucleation and initial growth stages of $\mathrm{ZrO}_{2}$ from zirconium n-propoxide on silicon (100) substrates (19). The results for 1-5 pulses showed how the high surface diffusivity and lack of nucleation sites on the silicon led to critical nucleation being achieved for large individual crystals before any evidence of deposition on the rest of the surface. The large individual crystals would form on any slight defect in the surface and were much larger than the nano-crystalite size detected in the film by TEM after several hundred pulses. None of the deposition investigations to-date shows evidence of carbon contamination from the precursor decomposition products. $\mathrm{Ta}_{2} \mathrm{O}_{5}$ was deposited from tantalum ethoxide at a rate up to 8 $\mu \mathrm{m} / \mathrm{hr}$ (20). Bioceramics are a current research topic with precursor development the main focus (21). Precursors for YBCO have been trialled, and work is continuing to develop a precursor recipe and processing parameters for the superconducting phase. 

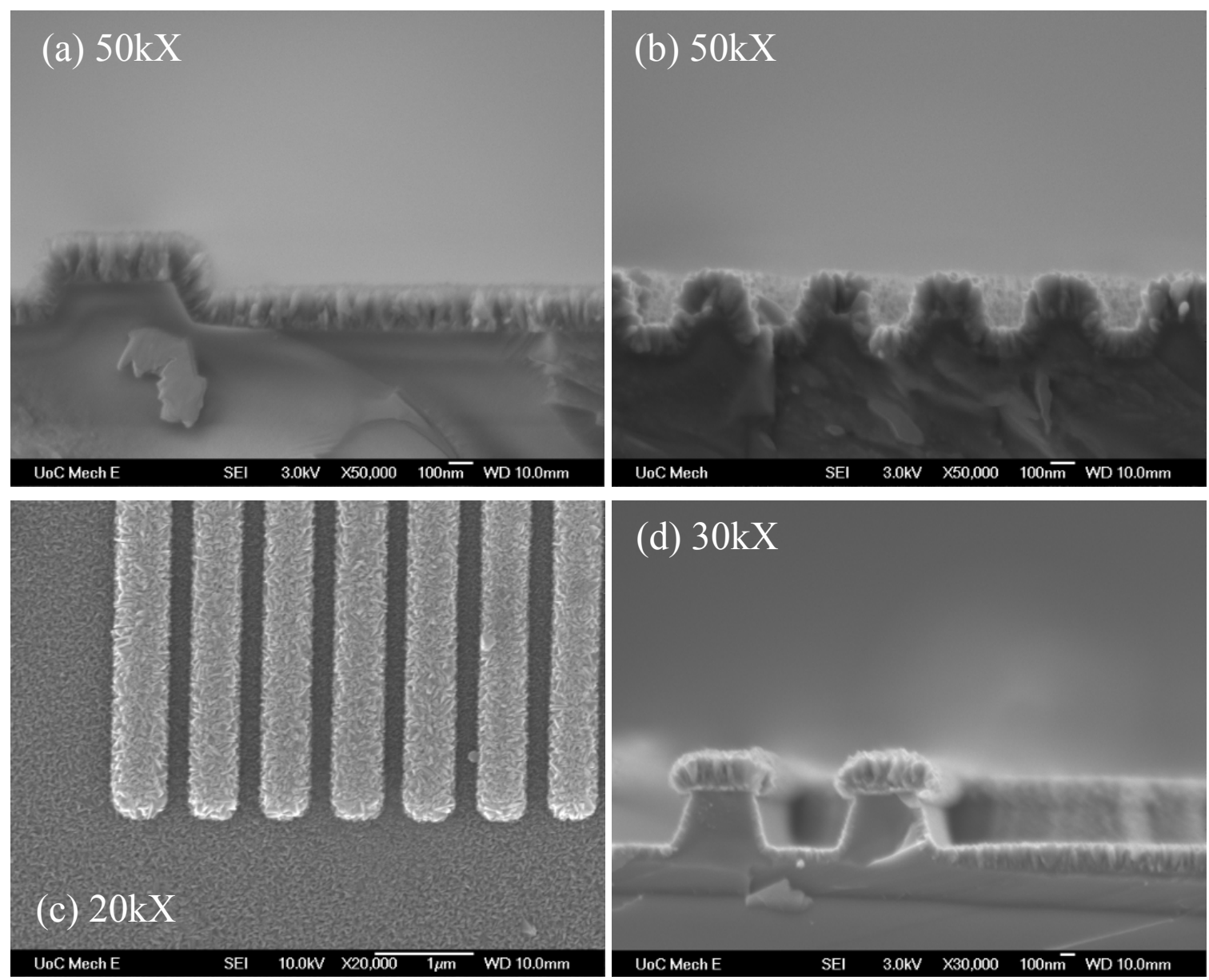

Figure 5. Nano-scale lithographed features in single crystal SiN were used as substrates for $\mathrm{TiO}_{2}$ deposition. (a) uniform layer on $300 \mathrm{~nm}$ width feature, (b) good uniformity on $100 \mathrm{~nm}$ trenches, (c) high arrival rate on $500 \mathrm{~nm}$ features produces uniform crystal structure with (d) crown accelerated growth with three times the thickness of the uniform trench coating of $60 \mathrm{~nm}$.

\section{Summary and Future Work}

Over the past ten years, the results of fundamental materials and flow dynamics research has led toward better understanding of the potential for the pulsed-pressure method of liquid injection MOCVD. The main conclusion is that pulsed-pressure MOCVD produces uniform vapor around the substrates and allows good control of the deposition process. The pulsed pressure concept has the interesting possibilities of low cost of the platform technology and the versatility of reactor design. The expansionregime flow is not sensitive to orientation and geometry, thus development times for new applications can be very short. Pulsed-pressure processing may not replace existing MOCVD tools, but it may open up possibilities for new applications and products. Current research includes working with industrial partners to develop PP-MOCVD tools for new applications in biomaterials for bone implants, thermal barrier coatings on casting forms, anti-corrosion coatings on marine energy components, self-cleaning container coatings, and anti-stick coatings on lithography moulds. 


\section{Acknowledgments}

I acknowledge Professor Rishi Raj for providing the challenge of low cost TBC's on microturbines by MOCVD which led to the technology developments described. I am grateful to the six PhD students, six Masters students, sixteen undergraduate students, my research partner Orfeo Sbaizero and two sabbatical visitors who have contributed to this work. Funding was provided by Industrial Research Ltd., the University of Canterbury, the National Science Foundation No. 9960174, the Royal Society of New Zealand Marsden Fund Grant UOC203, and the Foundation for Science Research and Technology UOCX0710. Nozzles were donated by Sono-Tek.

\section{References}

1. S. Krumdieck, in: Chemical Vapor Deposition: Precursors and Processes, A. Jones and M. L. Hitchman Editors, RSC Publishing, Cambridge, UK, p. 37 (2008).

2. S. Krumdieck, , O. Sbaizero, and R. Raj, Journal de Physique IV, 11 D. Davazoglou and C. Vahlas, Editors, EDP Sciences, France, 1161 (2001).

3. S.P. Krumdieck, J-Y Lee, and H. Raatz, in CVD-XVI/EUROCVD-14, M Allendorf, F. Maury and F. Teyssandier, Editors, PV 2003-08, p. 179, The Electrochemical Society Proceedings Series, Pennington, NJ (2003).

4. S. Krumdieck, Acta Materialia, 49583 (2001).

5. S. Krumdieck and R. Raj, Advanced Materials CVD, 7 No. 285 (2001).

6. S. Krumdieck, S.I. Baluti, L. Marcus, and A. Peled, in CVD-XVI/EUROCVD-15, A. Devi, R. Fischer, H. Parala, M. Allendorf, M. Hitchman, Editors, PV 2005-09, p. 120, The Electrochemical Society Proceedings Series, Pennington, NJ (2005).

7. F. Felten, J.P. Senateur, M. Labeau, K. Yu-Zhang, A. Abrutis, Thin Solid Films, 296 79 (1997).

8. H.M. Cave, K.-C. Tseng, J.-S. Wu, M.C. Jermy, J.-C. Huang, S.P. Krumdieck, J. Comp. Phys. 227 No. 12, 6249 (2008).

9. M.R. Smith, H.M. Cave, Y.-S. Chen, M.C. Jermy and J.-S. Wu, J. Comp. Phys. 228 (2009) 2213-2224.

10. S. Krumdieck, H.M. Cave, S. Baluti, M. Jermy, A. Peled, Chem. Eng. Sci., 62, 6121 (2007).

11. H.M. Cave, S. Krumdieck and M.C. Jermy, Chem. Eng. Journal, 135 1-2, 120 (2008).

12. H. Cave, M. Smith, J.-S. Wu, S. Krumdieck and M. Jermy, Proceedings. HPC Asia 2009, (2-5th March 2009, Kaohsiung, Taiwan).

13. S. Krumdieck and R. Raj, J. Amer. Ceram. Soc., 82[6], 1605 (1999).

14. S. Krumdieck and R. Raj, Surf. \& Coat. Tech., 141, 7 (2001).

15. V. Siriwongrungson, M. Alkaisi, S. Krumdieck, Surf. \& Coat. Tech., 201, 22-23, 8944 (2007).

16. Y. Akiyama, T. Sato, N. Imaishi, J. Crystal Growth, 147, 130 (1995).

17. S. Krumdieck, A. Kristinsdottir, L. Ramirez, M. Lebedev, N. Long, Surf. \& Coat. Tech., 201, 22-23, 8908 (2007).

18. S. Krumdieck, O. Sbaizero, A. Bullert and R. Raj, J. Amer. Ceram. Soc., 85[11], 2873 (2002).

19. L. Ramirez, M. Mecartney, S. Krumdieck, J. Mat. Res., 23 (8), 2202 (2008).

20. A. Porporati, S. Roitti, O. Sbaizero, J. Euro. Ceram. Soc., 23, 247 (2003).

21. R. Hartshorn, S. Stockwell, M. Lebedev, S. Krumdieck, Surf. \& Coat. Tech, 201, 2223, 9413 (2007). 